\title{
Pulmonary alveolar lipoproteinosis complicating juvenile dermatomyositis
}

\author{
M P SAMUELS, J O WARNER \\ From Brompton Hospital, London
}

ABSTRACT A 9 year old boy with juvenile dermatomyositis developed pulmonary alveolar lipoproteinosis. This previously unreported association was treated successfully by bronchoalveolar lavage.

Pulmonary alveolar lipoproteinosis is a rare condition of unknown aetiology characterised by accumulation of a lipoproteinaceous material within alveolar spaces. 'The diagnosis is confirmed by finding periodic acid-Schiff (PAS) staining material on light microscopy and characteristic lamellar bodies on electron microscopy. Although it has previously been reported to be associated with several immunological abnormalities in children and with haematological malignancies and respiratory infections in adults, an association with autoimmune disease has not been noted. We report a case of the disease associated with juvenile dermatomyositis.

\section{Case report}

Dermatomyositis was diagnosed in a 7 year old boy. He had a six month history of decreasing exercise tolerance and muscle stiffness and eventually developed a typical rash. At diagnosis his creatinine kinase was $534 \mathrm{IU} / 1$ and the antinuclear factor test gave a weakly positive result. He was treated with prednisolone, at a starting dose of $2 \mathrm{mg} / \mathrm{kg}$ daily. Progress was complicated by interfascial calcinosis and a fracture of his left humerus secondary to osteoporosis, but within two years he had nearly full muscle strength and the prednisolone had been reduced to less than $1 \mathrm{mg} / \mathrm{kg}$ on alternate days.

Two and a half years after the onset he developed a respiratory infection, for which he was treated with erythromycin; but he remained short of breath, had a persistent cough, and lost weight. A chest radiograph showed patchy infiltrates throughout both lung fields, but no hilar adenopathy, pleural effusion, or cardiomegaly. In view of the possibility of Pneumocystis carinii infection he received high dose co-trimoxazole. As his symptoms and radiographic findings did not improve he was referred for further investigation.

On admission his height and weight were on the 50th percentile. He had a heliotrope rash on his upper eyelids, generalised muscle wasting, and early clubbing. He was mildly cyanosed with a respiratory rate of $30 / \mathrm{min}$, poor chest expansion, and bilateral basal inspiratory crackles on auscul-

Address for reprint requests: Dr M P Samuels, Brompton Hospital, London SW3 6HP.

Accepted 28 Juby 1988 tation. Lung function tests showed reduced lung volumes with a severe mixed obstructive and restrictive defect and no response to a bronchodilator; transfer coefficient $(\mathrm{KCO})$ was normal at 1.92-2.35 mmol min $\mathrm{mPa}^{-1} \mathrm{l}^{-1}$.

His haemoglobin was $17.5 \mathrm{~g} / 1$ and his arterial oxygen tension $\left(\mathrm{PaO}_{2}\right) 5.5 \mathrm{kPa}$. Bronchial washings revealed no pathogens; they were not examined for PAS staining bodies. Open lung biopsy showed features typical of alveolar lipoproteinosis with no evidence of pulmonary fibrosis. Further investigations indicated normal white cell count, complement, immunoglobins, opsonins, and bone marrow. Lymphocyte subsets were normal, but there was a serum dependent defect of neutrophil candida phagocytosis and killing.

Bronchoalveolar lavage was performed in view of poor exercise tolerance, hypoxaemia, and an alveolar-arterial $\mathrm{Po}_{2}$ difference of $8 \mathrm{kPa}$. Under general anaesthesia with a double lumen endotracheal tube the right lung was lavaged with $500 \mathrm{ml}$ aliquots of normal saline, warmed to body temperature, until clear fluid returned. A total of 201 was used. The left lung was lavaged a week later. Both procedures were tolerated without complications.

There was an immediate symptomatic and physiological improvement and 12 months later exercise tolerance, lung function, and $\mathrm{PaO}_{2}$ were normal (table). The radiographic infiltrates have shown gradual clearing (figure). Muscle strength has remained normal as prednisolone has been slowly reduced.

\section{Discussion}

Pulmonary alveolar proteinosis is associated with immunological abnormalities, including hypogammaglobinaemia, lymphopenia, and thymic hypoplasia. ${ }^{2}$ Secondary infections, in particular with opportunist organisms, are often found and, with respiratory failure, contribute to the high mor-

\section{Results of hung function tests before and after} bronchoalveolar lavage

\begin{tabular}{|c|c|c|c|c|c|}
\hline & \multicolumn{2}{|c|}{ Before lavage } & \multicolumn{3}{|c|}{ After lavage } \\
\hline & $\begin{array}{l}\text { June } \\
1986\end{array}$ & $\begin{array}{l}\text { Oct } \\
1986\end{array}$ & $\begin{array}{l}\text { Nov } \\
1986\end{array}$ & $\begin{array}{l}\text { Jan } \\
1987\end{array}$ & $\begin{array}{l}\text { Apr } \\
1987\end{array}$ \\
\hline $\begin{array}{l}\text { Forced expiratory } \\
\text { volume in I s (ml) } \\
\text { Forced vital capacity (ml) } \\
\text { Total lung capacity (ml) }\end{array}$ & $\begin{array}{r}750 \\
870 \\
1920\end{array}$ & $\begin{array}{r}510 \\
940 \\
1590\end{array}$ & $\begin{array}{r}630 \\
980 \\
1900\end{array}$ & $\begin{array}{l}1020 \\
1370 \\
2140\end{array}$ & $\begin{array}{l}1450 \\
1650 \\
2110\end{array}$ \\
\hline $\begin{array}{l}\text { Airway resistance } \\
\text { (kPa 1-1 s) } \\
\text { Alveolar-arterial oxygen }\end{array}$ & 0.24 & 0.75 & 0.43 & 0.27 & 0.34 \\
\hline difference $(\mathbf{k P a})$ & & 8.9 & & 5.5 & $4 \cdot 1$ \\
\hline
\end{tabular}




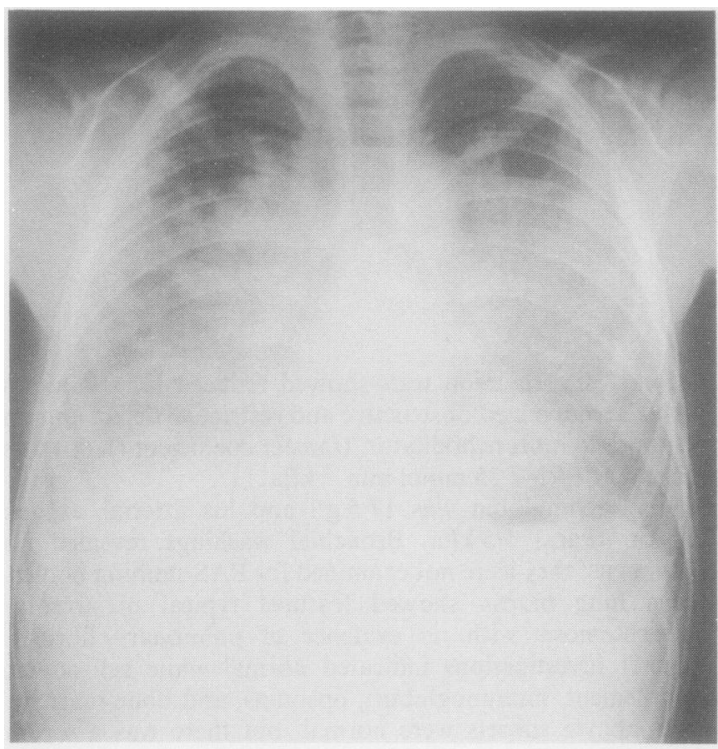

A

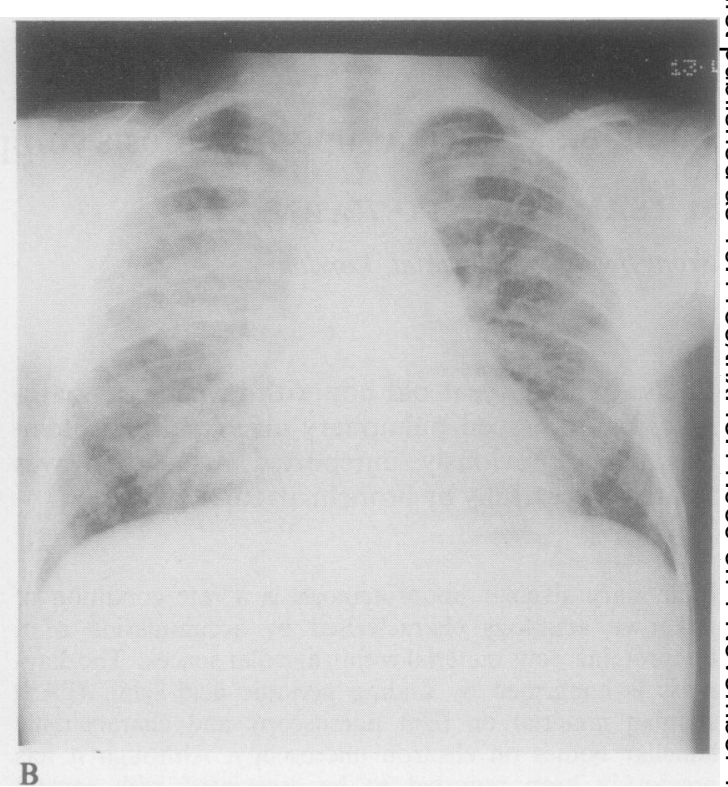

$\mathrm{B}$

Radiographic appearances before $(A)$ and after $(B)$ bronchoalveolar lavage.

tality. ${ }^{2}$ In adults it has been associated with haematological malignancy and silicosis. There has been a single report in association with autoimmunity but without clinically evident disease. ${ }^{3}$ A 3 year old girl had a low IgA concentration and a high positive smooth muscle antibody titre but no abnormal deposition of immunoglobulin in the lung was detected by fluorescence studies.

Our patient had clinically florid dermatomyositis with a serum dependent defect of neutrophil phagocytosis, but no immunoglobulin abnormalities. Lung biopsy showed no evidence of vasculitis or interstitial lung disease, although an intercostal muscle biopsy performed at the same time showed evidence of myositis. Whether the dermatomyositis has any bearing on the development of lipoproteinosis is questionable. Patients with alveolar lipoproteinosis have frequently either been taking corticosteroids at the onset or been treated with them. Patients having corticosteroids for other conditions, however, do not develop alveolar proteinosis. The initial trigger in our patient is likely to have been a respiratory infection, with either dermatomyositis, corticosteroid, or the serum defect (or a combination of these) causing an abnormal response in surfactant uptake. Abnormalities have been found both in type II alveolar cells and in alveolar macrophages, both of which are concerned in surfactant uptake and recycling.'

Spontaneous remission may occur in patients with pulmonary alveolar proteinosis, ${ }^{4}$ but bronchoalveolar lavage is indicated in those with appreciable dyspnoea, $\mathrm{PaO}_{2}$ less than $8.7 \mathrm{kPa}$, or an alveolar-arterial $\mathrm{Po}_{2}$ difference of more than $4.3 \mathrm{kPa}$. The primary effect of lavage is mechanical with immediate clinical and physiological improvement followed over several weeks by radiographic improvement. ${ }^{5}$ A single lavage may cause complete remission, as in our patient, suggesting that the primary abnormality is no longer present.

Lavage has sometimes been performed in small children and infants with cardiopulmonary bypass, to overcome hazards and technical difficulties. ${ }^{6}$ Fortunately, our patient was able to tolerate the smallest available double lumen endotracheal tube, though a double lumen Swan-Ganz catheter passed transbronchoscopically has been used in a smaller child. ${ }^{7}$ Whether this method or some form of extracorporeal circuit in infants and young children willo improve outcome is unknown.

Pulmonary alveolar proteinosis occurs in neonates with $\stackrel{\mathcal{D}}{\mathbb{D}}$ clinical and radiographic features similar to those of hyaline $\vec{\overrightarrow{ }}$ membrane disease. ${ }^{8}$ It may be a cause of chronic respiratory distress in both infants and older children. ${ }^{9}$ In the absence of $\widetilde{ }$ an infective agent, where immunocompetence is impaired产 this diagnosis should be considered.

\section{References}

1 Claypool WD, Rogers RM, Matuschak GM. Update on the clinical diagnosis, management and pathogenesis of pulmonary. alveolar proteinosis (phospholipidosis). Chest 1984;85:550-8.

2 Colon AR, Lawrence RD, Mills SD, O'Connell EJ. Childhoos pulmonary alveolar proteinosis. Report of a case and review of the literature. Am J Dis Child 1971;121:481-5.

3 Gray ES. Autoimmunity in childhood pulmonary alveolas proteinosis. Br Med J 1973;iii:296-7.

4 Kariman K, Kylstra JA, Spock A. Pulmonary alveolar protein osis: prospective clinical experience in 23 patients for 15 years Lung 1984;162:223-31.

5 Gale ME, Karlinsky JB, Robins AG. Bronchopulmonary lavage in pulmonary alveolar proteinosis: chest radiograph observa tions. Am J Radiol 1986;146:981-5.

6 Seard C, Wasserman K, Benfield JR, Cleveland RJ, Costley DO Heimlich EM. Simultaneous bilateral lung lavage (alveolato washing) using partial cardiopulmonary bypass. Report of two cases in siblings. Am Rev Respir Dis 1970;101:877-84.

7 Moazen F, Schmidt JH, Chesrown SE, et al. Total lung lavage for pulmonary alveolar proteinosis in an infant without the use of cardiopulmonary bypass. J Pediatr Surg 1985;20:398-401.

$8 \mathrm{Knight}$ DP, Knight JA. Pulmonary alveolar proteinosis in the newborn. Arch Pathol Lab Med 1985;109:529-31.

9 Coleman M, Dehner L, Sibley RK, Burke BA, L'Heureux PR, Thompson JR. Pulmonary alveolar proteinosis: an uncommor cause of chronic neonatal respiratory distress. Am Rev Respir Dis 1980;121:583-6. 